\title{
Preface
}

\section{Therapeutic Opportunities for Caffeine in Alzheimer's Disease and Other Neurodegenerative Disorders}

\author{
Alexandre de Mendonça ${ }^{a}$ and Rodrigo A. Cunha ${ }^{b}$ \\ anstitute of Molecular Medicine and Faculty of Medicine, University of Lisbon, Portugal \\ ${ }^{\mathrm{b}}$ Center for Neuroscience and Cell Biology of Coimbra and Faculty of Medicine, University of Coimbra, Portugal
}

\begin{abstract}
Although caffeine is the most widely consumed psychoactive drug worldwide, its potential beneficial effect for maintenance of proper brain functioning has only recently begun to be adequately appreciated. This has mainly resulted from the convergence of conclusions from epidemiological studies and from fundamental research in animal models. Epidemiological studies first revealed an inverse association between the chronic consumption of caffeine and the incidence of Parkinson's disease; this was paralleled by animal studies of Parkinson's disease showing that caffeine prevented motor deficits as well as neurodegeneration. Later a few epidemiological studies showed that the consumption of moderate amounts of caffeine was inversely associated with the cognitive decline associated with aging as well as the incidence of Alzheimer's disease. Again, this was paralleled by animal studies showing that chronic caffeine administration prevented memory deterioration and neurodegeneration in animal models of aging and of Alzheimer's disease.

This evidence was the driving force for a meeting on Caffeine and the Brain held in Lisbon on the 12-13 June 2009 joining several leading researchers dedicated to the effects of caffeine in the brain. Thanks to the generous sponsoring of Associação Industrial e Comercial do Café and to the efforts of its director, Margarida Ferreira, this gathering of clinical and fundamental researchers from very different disciplines discussed
\end{abstract}

issues ranging from molecular targets of caffeine, neurophysiological modifications and adaptations, to the potential mechanisms underlying the behavioral and neuroprotective actions of caffeine in distinct brain pathologies. Some consensual features emerged from the meeting, namely identifying adenosine $\mathrm{A}_{2 A}$ receptors as the main target for neuroprotection afforded by chronic caffeine consumption. In spite of the enthusiasm related to the multiple beneficial effects of caffeine for brain functioning, the greatest focus of the meeting was on the identification and discussion of several unresolved issues, which are hoped to set the stage for future research. Thus, issues of the mechanisms underlying caffeine neuroprotection remain unsolved, and in particular effects of caffeine on brain vasculature, on the blood brain barrier, on brain metabolism, and on neuroinflammation seem priority areas of research. While the potential of caffeine as a mood modifier was highlighted, particular attention was devoted to the impact of caffeine on cognition and memory performance. Caffeine seems particularly effective to normalize rather than bolstering memory performance and is a candidate disease-modifying agent for Alzheimer's disease, based on its neuroprotective profile and its ability to reduce amyloid- $\beta$ production. Although an inverse relationship between caffeine consumption and neurodegenerative disorders appeared compelling, it was consensual that several methodological issues must be solved before advancing to decisive clinical trials. 
In view of this current enthusiasm on the multiple beneficial effects of caffeine to normalize brain function and prevent its degeneration, we decided to propose to the Editors of Journal of Alzheimer's Disease organizing this special issue to convey the multiple views by different researchers on the roles of caffeine in the brain. Here we would like to thank George Perry and Mark A. Smith for supporting this endeavor, which is hoped to increase awareness of the potential benefits of caffeine. It is important to make it explicit that Ass- ociação Industrial e Comercial do Café generously financed both the meeting and this special issue while leaving full scientific independence to all contributors. Finally, we would like to thank all the participants, as well as the researchers that were invited and for several reasons declined to participate, since they were kind enough to give helpful suggestions and scientific comments that were ultimately reflected in this special issue of the Journal of Alzheimer's Disease.

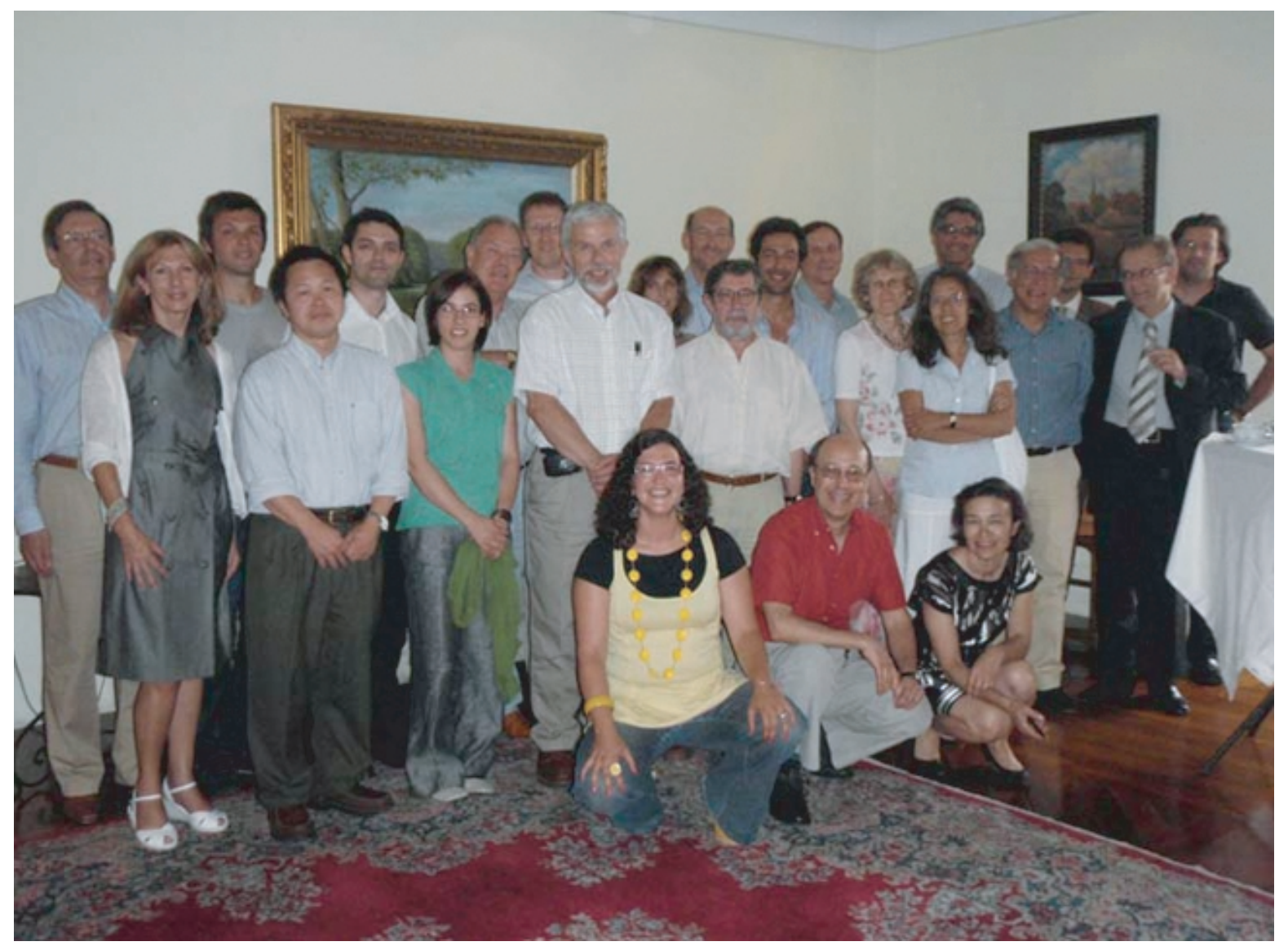

Participants in the meeting on Caffeine and the Brain held in Lisbon on the 12-13th June 2009. Standing, from the left to the right, Alexandre de Mendonça, Karen Ritchie, Florian Koppelstaetter, Jiang-Fan Chen, Vasco Sousa, Luísa Lopes, Lawrence Whalley, Geert Jan Biessels, Dale Pelligrino, Ana Rita Costenla, Jonathan Geiger, Joaquim Alexandre Ribeiro, Paulo Armada, Gary Arendash, Astrid Nehlig, Ana Sebastião, Rodrigo Cunha, Manuel Bicho, João Costa, Mamede de Carvalho, Nuno Lunet. Sitting, from the left to the right, Erica Marcelino, Marino Petracco, Margarida Ferreira. 